\title{
Early pain management after periodontal treatment in dogs - comparison of single and combined analgesic protocols
}

\author{
Petr Raušer ${ }^{1}$, Petr Janalík ${ }^{1}$, Martina Klimešová ${ }^{1}$, Magdaléna Marková ${ }^{1}$, Ladislav Stehlík², \\ Tomáš Fichtel ${ }^{1}$
}

University of Veterinary and Pharmaceutical Sciences Brno, Faculty of Veterinary Medicine, Small Animal Clinic, ${ }^{1}$ Department of Surgery and Orthopaedics, ${ }^{2}$ Department of Diagnostic Imaging, Brno, Czech Republic

Received July 18, 2014

Accepted May 13, 2015

\begin{abstract}
The aim of this study was to assess the analgesic effectiveness of three analgesic protocols in dogs undergoing a periodontal treatment. The study was performed as a prospective, randomized, "double blind" clinical study. A total of 45 client-owned dogs scheduled for periodontal treatment were included. Dogs of Group C received carprofen $\left(4 \mathrm{mg} \cdot \mathrm{kg}^{-1}\right)$, dogs of Group B received bupivacaine $\left(1 \mathrm{mg} \cdot \mathrm{kg}^{-1}\right)$ and dogs of Group $\mathrm{CB}$ received a combination of carprofen $\left(4 \mathrm{mg} \cdot \mathrm{kg}^{-1}\right)$ and bupivacaine $\left(1 \mathrm{mg} \cdot \mathrm{kg}^{-1}\right)$. Carprofen was administered subcutaneously $30 \mathrm{~min}$ before anaesthesia, bupivacaine was administered by nerve blocks in anaesthetized dogs. Painful periodontal treatment was performed in all patients, lasting up to one hour. Modified University of Melbourne Pain Score (UMPS), Visual Analogue Scale for pain assessment (VAS), plasma glucose and serum cortisol levels were assessed $30 \mathrm{~min}$ before administration of analgesics (C$0, \mathrm{~B}-0, \mathrm{CB}-0)$ and $2 \mathrm{~h}$ after recovery from anaesthesia (C-2, B-2, CB-2). For statistical analysis Friedman test, Mann-Whitney U-test, ANOVA and Fischer exact tests were used $(P<0.05)$. In $\mathrm{CB}-2$ compared to $\mathrm{CB}-0$ significantly decreased modified UMPS values. In CB-2 UMPS values were significantly lower compared to C-2 or B-2. In C-2 VAS values were significantly increased compared to $\mathrm{C}-0$, and in $\mathrm{B}-2$ VAS values were significantly increased compared to B-0. Visual Analogue Scale values were significantly lower in CB-2 compared to C-2 or B-2. Significantly increased plasma glucose concentrations were found in C-2 compared to C-0 and in B-2 compared to B-0. No other significant differences were detected. Administration of carprofen, bupivacaine or their combination is sufficient for early postoperative analgesia following periodontal treatment. Carprofen-bupivacaine combination is superior to carprofen or bupivacaine administered separately.
\end{abstract}

Dental pain, nerve block, teeth

In human as well as veterinary medicine, very painful pathological oral processes are dealt with frequently. Pain involves biochemical and pathological responses leading to the unpleasant sensation originating from tissue destruction. For maximum patient prosperity, individual, aggressive, and intense pain management is necessary. It can be achieved by preemptive and multimodal analgesic strategy (Beckman 2006; Rauser et al. 2013).

Preventive/preemptive analgesia, administration of analgesics prior to a painful stimulus, is more effective than giving the same drug thereafter (Muir 2009). Chronic painful conditions resulting from chronic periodontal disease should receive special consideration for preoperative pain management. Failure to provide preventive/preemptive analgesia makes postoperative pain management extremely difficult (Beckman 2006).

Multimodal pain management refers to the use of two or more analgesics from different classes in combination. This technique allows for a decrease in dosage for each of the agents, making the combination safer and more effective. This approach also allows the use of different analgesics to block pain in different nociceptive pathways. It should be noted that the local anaesthetics block pain in all three pathways of nociception (Beckman 2006).

Address for correspondence:

Petr Raušer

Small Animal Clinic, Faculty of Veterinary Medicine

University of Veterinary and Pharmaceutical Sciences Brno

Palackého tř. 1/3, 61242 Brno, Czech Republic 
Widely used and highly effective drugs for preventive/preemptive and multimodal analgesic approach are opioids, nonsteroidal anti-inflammatory drugs (NSAIDs), and local anaesthetics (Dzikiti et al. 2006). Analgesic mechanism of action of NSAIDs is inhibition of the inflammatory processes peripheral and central sensitization. They are anti-nociceptive both peripherally and centrally, independent of the anti-inflammatory effect. Non-steroidal anti-inflammatory drugs block neuronal plasticity and central sensitization (Vanegas and Tortorici 2002). A typical NSAID commonly used in small animal practice is carprofen.

Effective analgesia is provided by inhibition of sodium channels, complete blocking of the generation and conduction of nerve impulses induced by local nerve block techniques. Beside lidocaine, bupivacaine is one of the most widely used local anaesthetics in small animal practice. Compared to lidocaine, bupivacaine has slower onset of action ( $\sim 15 \mathrm{~min})$ and prolonged duration of effects (Woodward 2008).

This study was aimed at investigating the analgesic effects of carprofen, bupivacaine and their combination on early post-operative dental pain management. Effectiveness of the above mentioned analgesic combinations has not yet been published. This study develops previously published data oriented at analgesia of separately used drugs for dental pain management (Rauser et al. 2013).

\section{Materials and Methods}

All procedures were approved by the Animal Welfare Ethics Committee of the University of Veterinary and Pharmaceutical Sciences Brno and the present Code for Routine Clinical Procedures. All owners were informed of the planned procedure and signed an informed client consent, whereby they agreed with the extent of treatment and measurement.

Procedures did not exceed the standard range of routine clinical periodontal treatment.

The experimental protocol was adopted from our previous study comparing analgesic properties of carprofen, morphine, and bupivacaine after similar periodontal treatment in dogs (Rauser et al. 2013).

\section{Animals}

A total of 45 adult client-owned dogs of different breeds, 25 males and 20 females aged (mean \pm SD) $6.8 \pm$ 3.0 years, with a body mass of $8.1 \pm 5.9 \mathrm{~kg}$, were enrolled in this study. All dogs were clinically examined and blood samples for haematology and biochemistry were taken. Dogs were scheduled for periodontal treatment and did not have other health abnormalities. Dogs were allocated randomly to one of three groups - C (carprofen), B (bupivacaine) and CB (carprofen-bupivacaine combination), each containing 15 animals. All dogs were fasted overnight prior to anaesthesia, but had free access to water.

In all dogs, dental examination was performed and periodontal disease index (PDI, I-IV degree) was assessed.

The study was designed as a prospective, randomized, "double blind", controlled trial. Randomization was performed by drawing the group allocation.

\section{Anaesthesia}

Venous catheter was placed in the cephalic vein in all dogs. The dogs were sedated with medetomidine (0.005 mg $\mathrm{kg}^{-1}$ i.v.; Domitor $1 \mathrm{mg} / \mathrm{ml}$, Pfizer, Finland). Thereafter, anaesthesia was induced with propofol (1-3 mg $\mathrm{kg}^{-1}$ i.v.; Norofol $10 \mathrm{mg} / \mathrm{ml}$, Norbrook, North Ireland) and the dogs were orotracheally intubated. Anaesthesia was maintained with isoflurane (1.5-2.0 vol.\%, Isofluran, Torrex Chiesi, Czech Republic) in oxygenair by semi-closed anaesthetic circuit with spontaneous breathing (Cavendish 500, MIE). Pulse rate, respiratory rate, end-tidal $\mathrm{CO}_{2}$ and oxygen saturation of haemoglobin were monitored (LiveSense, Medair). Depth of anaesthesia was assessed by painful stimulation response.

At the end of the periodontal treatment, medetomidine was reversed by atipamezole $\left(0.0125 \mathrm{mg} \cdot \mathrm{kg}^{-1} \mathrm{IM}\right.$; Antisedan $5 \mathrm{mg} / \mathrm{ml}$, Orion Pharma, Finland) administered intramuscularly (i.m.).

\section{Analgesia}

Dogs of Group C $(\mathrm{n}=15)$ received carprofen $\left(4 \mathrm{mg} \cdot \mathrm{kg}^{-1}\right.$; Rimadyl, Pfizer, Belgium), dogs of Group B $(\mathrm{n}=15)$ received bupivacaine $\left(1 \mathrm{mg} \cdot \mathrm{kg}^{-1}\right.$; Marcaine $0.5 \%$, Astra Zeneca, U.K.) and dogs of Group CB $(\mathrm{n}=15)$ received a carprofen and bupivacaine combination.

Thirty min before sedation carprofen was administered subcutaneously (s.c.) in animals of groups C and CB. In anaesthetized dogs of groups B and CB, bilateral nerve blocks of maxillary and alveolar inferior nerves were performed by intraoral injection technique (Beckman 2013) with bupivacaine. All nerve blocks were performed by a single person (Rauser). 
Procedure

In all dogs, PDI levels were assessed (Wiggs and Lobprise 1997) and recorded. Periodontal treatment started 15 min after nerve block loading time. Invasive subgingival scaling was performed on all teeth in all 45 dogs. The total periodontal treatment lasted up to one hour. All procedures were performed by the same dentist (Janalik).

Pain assessment

Modified University of Melbourne Pain Score (UMPS, range 0-28 points), Visual Analogue Scale for pain assessment (VAS, range 0-100 mm), plasma glucose (Glu) and serum cortisol (Cor) concentrations were assessed before administration of analgesics $30 \mathrm{~min}$ before anaesthesia (C-0, B-0, CB-0), $2 \mathrm{~h}$ thereafter, and $30 \mathrm{~min}$ after atipamezole administration (C-2, B-2, CB-2).

For modified UMPS assessment, breathing frequency ( $0-3$ points), heart rate $(0-3)$, body temperature $(0-1)$, pupil size $(0-2)$, salivation ( $0-2)$, palpation response $(0-2)$, jaw tremor $(0-2)$, jaw self-mutilation $(0-2)$, vocalization (0-2), mental status ( $0-3)$, spontaneous behaviour ( $0-3)$ and manipulation response $(0-3)$ were used.

For VAS assessment "blind" analgesiometic ruler (Janssen-Cillag, 0-100 mm) was used. Blood samples for determination of plasma glucose and serum cortisol concentrations were collected from the jugular vein. Only animals with plasma glucose concentrations of 3.1-6.7 mmol. $\mathrm{l}^{-1}$ and serum cortisol concentrations of $27.6-165.5 \mathrm{nmol} \cdot 1^{-1}$ were included in our study.

All modified UMPS, VAS or blood sample collections were performed by a single person (Klimesova), who was blinded to the analgesics administered. Specific nerve block side-effects (exophthalmos, automutilation, jaw tremor, tongue paresis, changes of pupil size, seizures, blindness) were recorded.

Rescue analgesia

Rescue analgesia with tramadol ( $2 \mathrm{mg} \cdot \mathrm{kg}^{-1}$ i.m.; Tramadol AL $100 \mathrm{mg}$, Alind Pharma) was provided at UMPS of 14 points or greater, and at VAS of $50 \mathrm{~mm}$ or greater. In patients needing rescue analgesia, VAS or modified UMPS values were re-evaluated every hour and tramadol was re-administered.

\section{Statistical analysis}

Data normality was checked using Shapiro Wilk test and, according to these test results, differences in plasma glucose and serum cortisol concentrations between and within groups were analysed using analysis of variance (ANOVA) for repeated measures. Modified UMPS and VAS score differences over time for each group were determined using Friedman test, while the group comparisons were done pre- and postoperatively using MannWhitney U-test. Nerve block specific side-effects and the number of patients requiring rescue analgesia in each group were assessed using Fischer exact test.

Statistical analyses were performed using the commercially available software SPSS and Microsoft Excel. Differences were considered significant at $P<0.05$.

\section{Results}

There were no significant differences between groups with respect to sex, body mass $($ mean $\pm \mathrm{SD}$ ) (Group C $7.0 \pm 4.4 \mathrm{~kg}$, Group B $9.1 \pm 4.2 \mathrm{~kg}$, Group CB $8.3 \pm 4.4 \mathrm{~kg}$ ), age (Group C $6.6 \pm 3.2$ years, Group B $5.7 \pm 1.8$ years, Group CB $8.1 \pm 3.5$ years), treatment time (Group C $42 \pm 5 \mathrm{~min}$, Group B $40 \pm 7 \mathrm{~min}$, Group CB $45 \pm 5 \mathrm{~min}$ ) or PDI level (Group C $2 \pm 1$, Group B $2 \pm 1$, Group CB $3 \pm 1$ ).

Modified UMPS post-treatment values (Table 1) decreased significantly in Group CB compared to pre-treatment values $(P=0.001)$. Modified UMPS values were significantly lower after treatment in Group CB compared to Group C $(P=0.004)$ or Group B $(P=$ 0.048).

Visual Analogue Scale post-treatment values (Table 1) increased significantly from the baseline in Group C $(P=0.007)$ and in Group B $(P=0.027)$. Visual Analogue Scale values were significantly lower after treatment in Group $\mathrm{CB}$ when compared to Group $\mathrm{C}$ $(P=0.004)$ or Group B $(P=0.011)$.

Plasma glucose concentrations (Table 1$)$ increased significantly after the treatment in Group C $(P=0.029)$ and in Group B $(P=0.030)$. No significant differences in posttreatment plasma glucose concentrations were detected between groups.

No significant differences in serum cortisol concentrations within or between groups were detected.

Rescue analgesia was necessary in one patient of Group C only (VAS $=50 \mathrm{~mm}$ ). No follow-up rescue analgesia was needed. Side effects associated with the nerve block were 
Table 1. Data of the Visual Analogue Scale (VAS), modified University of Melbourne Pain Score (UMPS), plasma glucose and serum cortisol concentrations are expressed as mean $\pm \mathrm{SD}$.

\begin{tabular}{|c|c|c|c|c|c|c|}
\hline & UMPS (points) & VAS (mm) & $\begin{array}{l}\text { Plasma glucose } \\
\left(\left(\mathrm{mmol} \cdot 1^{-1}\right)\right.\end{array}$ & $\begin{array}{l}\text { Serum cortisol } \\
\quad\left(\mathrm{nmol} \cdot \mathrm{l}^{-1}\right)\end{array}$ & $\begin{array}{c}\text { Rescue analgesia } \\
\text { (number } \\
\text { of patients) }\end{array}$ & $\begin{array}{c}\text { Nerve block } \\
\text { complication } \\
\text { (number } \\
\text { of patients) }\end{array}$ \\
\hline Group C-0 & $2 \pm 2$ & $11 \pm 8$ & $6.1 \pm 0.5$ & $107.4 \pm 45.7$ & & \\
\hline Group C-2 & $3 \pm 2$ & $24 \pm 13^{b}$ & $6.9 \pm 1.2^{\mathrm{b}}$ & $137.8 \pm 94.3$ & 1 & \\
\hline Group B-0 & $2 \pm 1$ & $11 \pm 7$ & $5.9 \pm 0.5$ & $114.5 \pm 39.5$ & & \\
\hline Group B-2 & $2 \pm 2$ & $18 \pm 9^{b}$ & $6.5 \pm 1.3^{\mathrm{b}}$ & $111.5 \pm 111.4$ & & 5 \\
\hline Group CB-0 & $4 \pm 2$ & $12 \pm 5$ & $5.8 \pm 0.8$ & $114.9 \pm 48.3$ & & \\
\hline Group CB-2 & $2 \pm 1^{\mathrm{ac}}$ & $9 \pm 3^{c}$ & $6.4 \pm 1.9$ & $96.2 \pm 58.3$ & & 1 \\
\hline
\end{tabular}

a - significant decrease compared to pre-treatment values (CB-2 vs. CB-0)

b - significant increase compared to pre-treatment values (C-2 vs. C-0 and B-2 vs. B-0)

c - significant decrease in CB-2 compared to C-2 and to B-2

observed in 5 dogs of Group B ( 2 dogs had mild unilateral exophthalmos - protrusion of eye globe from orbit compared to contralateral eye, 3 dogs jaw self-mutilation) and in 1 dog of Group CB (jaw self-mutilation). Exophthalmos disappeared in both Group B dogs within one hour without any notable discomfort or emergency medication requirement. Jaw self-mutilation was mild, occurring $10 \pm 2$ min after extubation and resolved spontaneously upon complete recovery. No treatment or sedation was required. No significant differences between groups were detected at the end of the study period.

\section{Discussion}

Dental pain assessment has an important influence on the results of this study. A precise algorithm for specific dental pain has not been published. A study by Rauser et al. (2013) suggested a dental pain assessment algorithm based on modified UMPS and VAS algorithm and glucose and cortisol concentrations (Martins et al. 2010), used in this study, too. Measurement of changes in amplitude of reflex-evoked muscle action potentials was described by Brown et al. (2002). However, this measurement is not available in clinical practice for client-owned patients.

Increased glucose concentrations could be caused by medetomidine (Burton et al. 1997). We used the same dose of medetomidine in all patients and the dose was low. Therefore, the effect of medetomidine on glucose concentrations should not be significant and could not influence the definitive pain assessment. The cortisol plasma concentration is not influenced by medetomidine administration (Ko et al. 2000). Moreover, a similar dose of medetomidine was administered in all groups. Therefore, the risk of associated error should be comparable in all groups.

For preemptive analgesia, dental procedures including, NSAIDs and local anaesthetics are very popular. The goal of this study was to investigate their efficacy when used separately or in combination. We used the NSAIDs carprofen and long acting local anaesthetic lidocaine separately and in combination. Only Locher-Claus et al. (2005) and Rauser et al. (2013) published a comparable analgesic approach of similar analgesic agents used for postoperative dental analgesia in animals (rats and dogs); however, they used all the analgesics only separately.

Locher-Claus et al. (2005) compared morphine, ibuprofen, and bupivacaine before pulp exposure, which induced dental pain. Their results suggest that preemptive morphine 
or bupivacaine treatment can significantly decrease postoperative pain compared to ibuprofen administration. Results of their study differ from the results of our study. In dogs undergoing the periodontal treatment, analgesia induced with carprofen was more effective when compared to morphine. This variance could have been caused by the markedly higher dose of morphine used by Locher-Claus et al. (2005).

Rauser et al. (2013) compared morphine, carprofen, and bupivacaine for early postoperative analgesia (up to $2 \mathrm{~h}$ ) following sub-gingival scaling for the periodontal treatment in dogs. Bupivacaine nerve blocks could be superior to carprofen, which could be superior to morphine. Results of the study are similar with the present study, because similar analgesic protocol and pain assessment were used.

We recorded significantly decreased UMPS values in $\mathrm{CB}-2$ when compared to CB-0, lower VAS values in CB-2 compared to C-2 and B-2 and increased plasma glucose concentrations in $\mathrm{C}-2$ compared to $\mathrm{C}-0$ and $\mathrm{B}-2$ to $\mathrm{B}-0$. We did not detect a decrease of other analysed blood values. Based on these facts, it is not possible to state that an individual drug or the combination used is markedly superior to either drug alone; however, the clinical pain assessment (UPMS, VAS) suggests that the analgesic effect of the combination of carprofen and bupivacaine is moderately better. It should confirm the multimodal analgesic theory (Beckman 2006) as the local anaesthetic bupivacaine works on a different mechanism than the NSAID carprofen. Their reciprocal analgesic effects are combined. Beckman (2013) recommends the combination of local anaesthetics and NSAIDs for multimodal pain management in mild to moderate pain states. However, in moderate to severe pain states, an opioid and gabapentin should be added.

Because of the subjective pain assessment model, UMPS and VAS values showed a wide range and image variability of this method of assessment, which can influence the results. In Group CB modified UMPS values were significantly decreased. This could occur due to an inaccurate method of dental pain assessment. The UMPS values in Group B and Group CB could be biased, because some animals manifested self-mutilation of the associated oral region after bupivacaine administration due to insensitivity induced by the nerve block and not due to pain. The same results were presented by Rauser et al. (2013) in animals, in which bupivacaine was used. Non-significant changes could be caused by the short examination interval between the second pain assessment and the end of anaesthesia. The anaesthetics (residual effects) could work despite the antagonisation of sedatives (medetomidine).

Pain intensity can be associated with increase of plasma glucose or serum cortisol concentrations as a stress response. Changes of the above mentioned biochemical indicators may offer more information about analgesic efficacy (Martins et al. 2010). In the present study, blood glucose concentrations were significantly increased after carprofen or bupivacaine administration compared to the pre-treatment values. Pre-treatment and post-treatment blood glucose concentrations after carprofen-bupivacaine combination were without significant changes. This could indicate inferior analgesia after carprofen or bupivacaine alone in comparison to the drug combination. In our study we detected a non-significant increase in serum cortisol concentrations after administration of carprofen alone and a non-significant decrease after administration of bupivacaine or carprofenbupivacaine combination. These changes could indicate the quality of analgesia. However, differences in serum cortisol concentrations were non-significant.

Insufficient postoperative analgesia was detected in one patient of the carprofen group only. This fact confirms that all analgesic protocols and methods provide adequate analgesia in dogs after periodontal treatment. The nerve block by bupivacaine evokes a complete block of nociception and transmission; therefore its analgesic effect should be the best. The effects of the NSAID carprofen are central and peripheral action (Yaksh and Malmberg 1993) and antiphlogistic activity too. Common consequences of periodontal disease are 
soft tissue inflammatory processes - stomatitis and gingivitis, which are inhibited by carprofen. The influence of carprofen, its analgesic and antiphlogistic actions could have a more intense effect.

Carprofen administered alone is efficient for the management of mild to medium pain intensity (Beckman 2013), whereas the complete nerve block provided by bupivacaine should reduce pain of different intensities from mild to severe. Periodontal treatments performed within our study caused mild to medium pain. The analgesic effects of carprofen did not need to be automatically outperformed by local anaesthetics.

In human dentistry, many studies focusing on the efficacy of NSAIDs for dental pain management have been published. These drugs represent the gold analgesic standard (Huber and Terezhalmy 2006; Rauser et al. 2013).

The non-steroidal anti-inflammatory drug carprofen does not show a significant difference in analgesia compared to bupivacaine or combination of both. It can be therefore considered a drug of choice for dental analgesia in dogs, as well.

Pain management of postoperative dental pain with NSAIDs is generally equivalent or superior to opioids in musculoskeletal pain management (Becker 2010). Dental pain is included in the musculoskeletal category.

With regard to the duration of the analgesic action, bupivacaine produces sufficient analgesia for $3 \mathrm{~h}$ for pulpal analgesia and up to $9 \mathrm{~h}$ for periodontal tissue analgesia (Gracis 2013). Carprofen eases pain for 12-24 h (Dyson 2008). The combination of both drugs should produce excellent analgesia for dental pain for 3-9 h depending on the dental procedure.

The administration of carprofen, bupivacaine or the carprofen-bupivacaine combination is sufficient for early postoperative analgesia following the periodontal treatment in dogs. However, the results of this study indicate that the carprofen-bupivacaine combination should be superior to carprofen or bupivacaine administered alone.

\section{Acknowledgement}

This work was supported by the Ministry of Education, Youth and Sports (Research Project IGA VFU No. 60/2012/FVL).

\section{References}

Becker DE 2010: Pain management: Part 1: Managing acute and postoperative dental pain. Anesth Prog 57: 67-79

Beckman BW 2006: Pathophysiology and management of surgical and chronic oral pain in dogs and cats. J Vet Dent 23: 50-60

Beckman B 2013: Anesthesia and pain management for small animals. Vet Clin North Am Small Anim Pract 43: 669-688

Brown DC, Bernier N, Shofer F, Steinberg SA, Perkowski SZ 2002: Use of non-invasive dental dolorimetry to evaluate analgesic effects of intravenous and intrathecal administration of morphine in anesthetized dogs. Am J Vet Res 63: 1349-1353

Burton SA, Lemke KA, Ihle SL, Mackenzie AL 1997: Effects of medetomidine on serum insulin and plasma glucose concentrations in clinically normal dogs. Am J Vet Res 58: 1440-1442

Dyson D 2008: Analgesia and chemical restraint for the emergent veterinary patient. Vet Clin North Am Small Anim Pract 38: 1329-1352

Dzikiti TB, Joubert KE, Venter LJ, Dzikiti LN 2006: Comparison of morphine and carprofen administered alone or in combination for analgesia in dogs undergoing ovariohysterectomy. J S Afr Vet Assoc 77: 120-126

Gracis M 2013: The oral cavity. In: Campoy L, Read MR (eds): Small animal regional anesthesia and analgesia, 1st edn. Wiley-Blackwell, Ames, pp. 119-140

Huber MA, Terezhalmy GT 2006: The use of COX-2 inhibitors for acute dental pain: A second look. J Am Dent Assoc 137: 480-487

Ko JC, Mandsager RE, Lange DN, Fox SM 2000: Cardiorespiratory responses and plasma cortisol concentrations in dogs treated with medetomidine before undergoing ovariohysterectomy. J Am Vet Med Assoc 15: 217, 509-514

Locher-Claus MT, Erickson TE, Law AS, Johnson WT, Gebhart GF 2005: Effects of preemptive morphine, ibuprofen or local anesthetic on Fos expression in the spinal trigeminal nucleus following tooth pulp exposure in the rat. J Endod 31: 578-583 
Martins LT, Kahvegian MAP, Noel-Morgan J, Leon-Roman MA, Otsuki DA, Fantoni DT 2010: Comparison of the effects of tramadol, codeine, and ketoprofen alone or in combination on postoperative pain and on concentrations of blood glucose, serum cortisol, and serum interleukin-6 in dogs undergoing maxillectomy or mandibulectomy. Am J Vet Res 71: 1019-1026

Muir WW 2009: Physiology and pathophysiology of pain. In: Gaynor JS, Muir WW (Eds): Handbook of veterinary pain management, $2^{\text {nd }}$ edn. Mosby, St. Louis, pp. 13-45

Rauser P, Janalik P, Markova M, Fichtel T 2013: Early analgesia after periodontal treatment in dogs: a comparison of three analgesic protocols. Vet Med Czech 58: 312-317

Vanegas H, Tortorici V 2002: Opioidergic effects of nonopioid analgesics on the central nervous system. Cell Mol Neurobiol 22: 655-661

Woodward TM 2008: Pain management and regional anesthesia for the dental patient. Top Companion Anim Med 23: 106-114

Yaksh TL, Malmberg AB 1993: Spinal actions of NSAIDs in blocking spinally mediated hyperalgesia: the role of cyclooxygenase products. Agents Actions Suppl 41: 89-100

Wiggs RB, Lobprise HB 1997: Periodontology. In: Wiggs RB, Lobprise HB (Eds): Veterinary dentistry. Principles and Practice, $1^{\text {st }}$ edn. Lipicott - Raven, Philadelphia - New York, pp. 186-231 